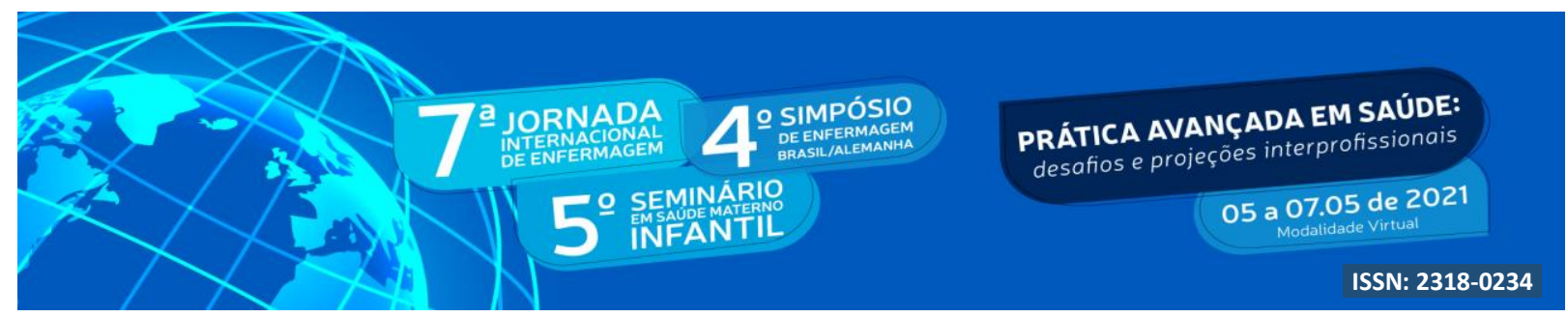

DOI: http://doi.org/10.48195/jie2021-063

\title{
A CONSTRUÇÃO DE UM CONCEITO DE SAÚDE AMPLIADO E INÉDITO. ${ }^{1}$
}

\section{Caroline de Oliveira Salvati²; Gustavo Salvati ${ }^{3}$; Bruna Ruoso da Silva Neutzling ${ }^{4}$ Rosemary Silva Silveira ${ }^{5}$; Dirce Stein Backes ${ }^{6}$}

\begin{abstract}
RESUMO
Objetivo: construir um conceito de saúde ampliado e inédito, a partir de leituras individuais e interações grupais, conforme proposto pela disciplina Conceito de Saúde e seus Nexos no Trabalho da Enfermagem/Saúde do Mestrado em Enfermagem (PPGEnf/FURG). Métodos: artigo teórico-reflexivo elaborado a partir das atividades digitais síncronas, realizadas durante a pandemia mundial de COVID-19 entre os meses de agosto e outubro de 2020. Resultados: foi elaborado um conceito de saúde ampliado e inédito, embasado nas leituras propostas pela disciplina de Conceito de Saúde e seus Nexos no Trabalho da Enfermagem/Saúde. Conclusão: o estudo possibilitou o processo de construção de um conceito de saúde ampliado e inédito, abrangente em relação aos contextos sociais, políticos, econômicos, ambientais e singulares. Observa-se ainda que a reflexão sobre o conceito de saúde, objetivando a ampliação do olhar em relação aos seus determinantes pode contribuir para práticas profissionais de atenção integral à saúde das pessoas.
\end{abstract}

Palavras-chave: Conceito de saúde; Enfermagem; Saúde.

\section{ABSTRACT}

Objective: to build an expanded and unprecedented concept of health, based on individual readings and group interactions, as proposed by the discipline Health Concept and its Nexuses in Nursing Work / Health of the Master of Nursing (PPGEnf / FURG). Methods: theoreticalreflective article elaborated from the synchronous digital activities, carried out during the world pandemic of COVID-19 between the months of August and October 2020. Results: an

\footnotetext{
${ }^{1}$ Artigo teórico-reflexivo elaborado a partir das orientações da disciplina de Conceito de Saúde e seus Nexos no Trabalho da Enfermagem/Saúde, do Programa de Pós-graduação em Enfermagem da Universidade Federal do Rio Grande (PPGEnf/FURG).

${ }^{2}$ Doutoranda em Enfermagem do Programa de Pós-Graduação em Enfermagem da Universidade Federal de Rio Grande. E-mail: enf.carolinedeo@gmail.com

${ }^{3}$ Médico. Especialista. E-mail: gustavosalvati@yahoo.com

${ }^{4}$ Doutoranda em Enfermagem do Programa de Pós-Graduação em Enfermagem da Universidade Federal de Rio Grande. E-mail:brunaneutzling10@gmail.com

${ }^{5}$ Orientadora. Doutora. Programa de Pós-Graduação em Enfermagem da Universidade Federal de Rio Grande. E-mail: rosemaryssilveira@gmail.com

${ }^{6}$ Orientadora. Doutora. Mestrado Profissional em Saúde Materno Infantil. Universidade Franciscana. Santa Maria/RS. E-mail: backesdirce@ufn.edu.br
} 


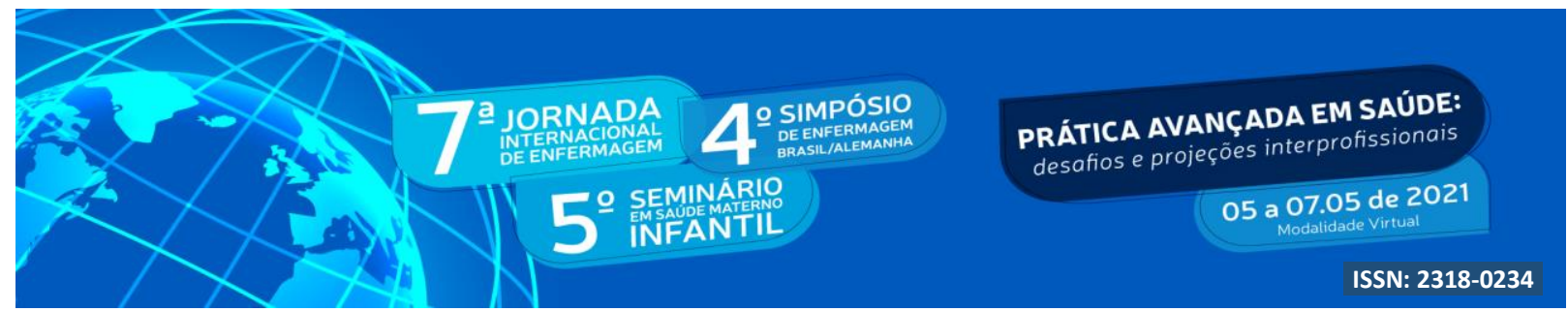

expanded and unprecedented concept of health was elaborated, based on the readings proposed by the discipline of Health Concept and its Nexuses in Nursing / Health Work. Conclusion: the study enabled the process of building a broad and unprecedented concept of health, comprehensive in relation to social, political, economic, environmental and singular contexts. It is also observed that the reflection on the concept of health, aiming at broadening the look in relation to its determinants, can contribute to professional practices of comprehensive health care for people.

Keywords: Community; Family; Nursing.

\section{INTRODUÇÃO}

A saúde conceituada como um estado de completude nas esferas física, mental e social, é a proposição adotada pela Organização Mundial de Saúde (WHO, 1948) e largamente difundida, porém com as enormes iniquidades sofridas pela população mundial na atualidade, esse estado de completo bem-estar se torna inatingível e impossível de ser mantido de forma integral. Já a saúde entendida como um direito universal e que deve ser garantida por meio de políticas e programas que diminuam as desigualdades, que é um conceito que está implícito na Constituição Federal Brasileira (BRASIL, 1988), sendo uma ideia que inspira o desenvolvimento das ações e serviços do Sistema Único de Saúde e de forma prática, colabora para devolução da dignidade e humanidade às pessoas (SCLIAR, 2007).

E a manutenção da dignidade do indivíduo está condicionada à sua capacidade de ser autônomo e prover as suas necessidades básicas através do seu trabalho. A capacidade de trabalhar e construir ferramentas é o que difere o homem moderno do homem primitivo, assim, os aspectos relacionados ao trabalho afetam a saúde das pessoas, bem como as condições sociais para a realização do trabalho que são determinadas pelo meio. Portanto a capacidade laboral das pessoas é um dos determinantes no binômio saúde-doença (MARX, 2017).

Faz-se necessário discutir o estado de doença, a partir da retirada do estado de saúde como completude absoluta, caminhando para a ampliação do olhar para o conceito de saúde. Para tanto, Canguilhem (2012) descontrói a ideia de que saúde e normalidade são sinônimas, demonstrando a partir do seu referencial teórico que tanto a saúde quanto a doença são estados de normatividade. Quando a pessoa se sente saudável ela está de acordo com a sua 


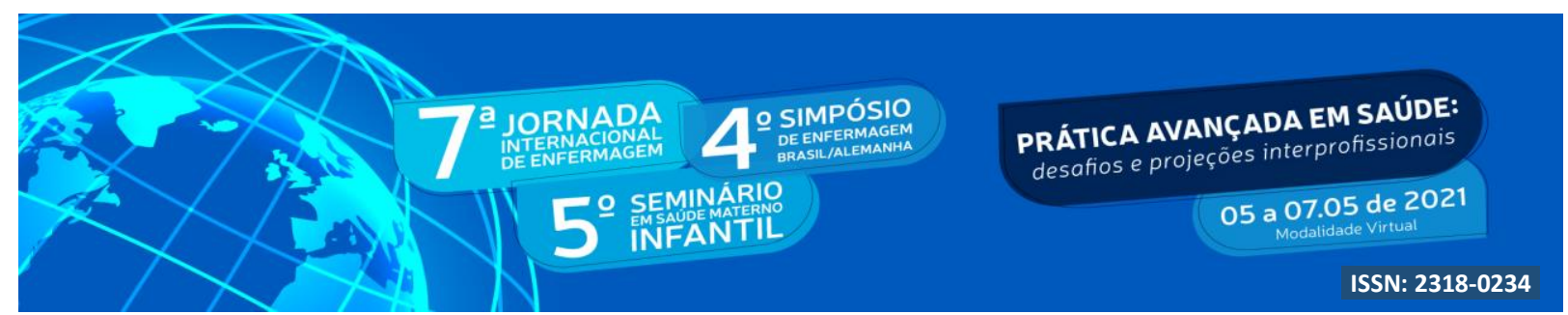

norma biológica estabelecida no momento e quando uma doença abala essa norma, o corpo humano tem a capacidade de reestabelecer uma nova norma, diferente da anterior, mas com a mesma capacidade de adaptação às intempéries da vida. Dessa forma, conforme o autor, saúde e doença são estado de adaptação ao meio.

Nesse sentido, saúde e doença tem representatividade diferente aos indivíduos, o patológico para um pode ser a normatividade de outro (CANGUILHEM, 2012). Esses conceitos apreendem sentido, conforme inúmeros fatores, tais como as concepções individuais e científicas, época, local, classe, social (SCLIAR, 2007). Diante do exposto, justifica-se a necessidade de aprofundar os estudos referentes ao conceito de saúde, buscando alternativas mais abrangentes e ampliadas, que abarquem as singularidades das pessoas e coletividades, ambasando práticas profissionais afinadas a atenção integral à saúde das pessoas.

\section{OBJETIVO}

Construir um conceito de saúde ampliado e inédito, a partir de leituras individuais e interações grupais, conforme proposto pela disciplina Conceito de Saúde e seus Nexos no Trabalho da Enfermagem/Saúde do Mestrado em Enfermagem (PPGEnf/FURG).

\section{METODOLOGIA}

Trata-se de um artigo teórico-reflexivo, desenvolvido como trabalho final pela disciplina de Conceito de Saúde e seus Nexos no Trabalho da Enfermagem/Saúde, do Programa de Pós-Graduação em Enfermagem (PPGEnf/FURG). A disciplina foi desenvolvida na perspectiva da Educação online, com um conjunto de conteúdos e situações estruturados no formato de tecnologias digitais síncronas e assíncronas, no período de agosto a outubro de 2020, frente à situação da pandemia da COVID-19. O conjunto de atividades foi constituído de: leituras de textos; reflexões escritas; discussões; elaboração de exercícios teóricos a partir das leituras interpretadas e compreendidas; elaboração de um artigo final com o intuito de construir um conceito de saúde ampliado e inédito. 


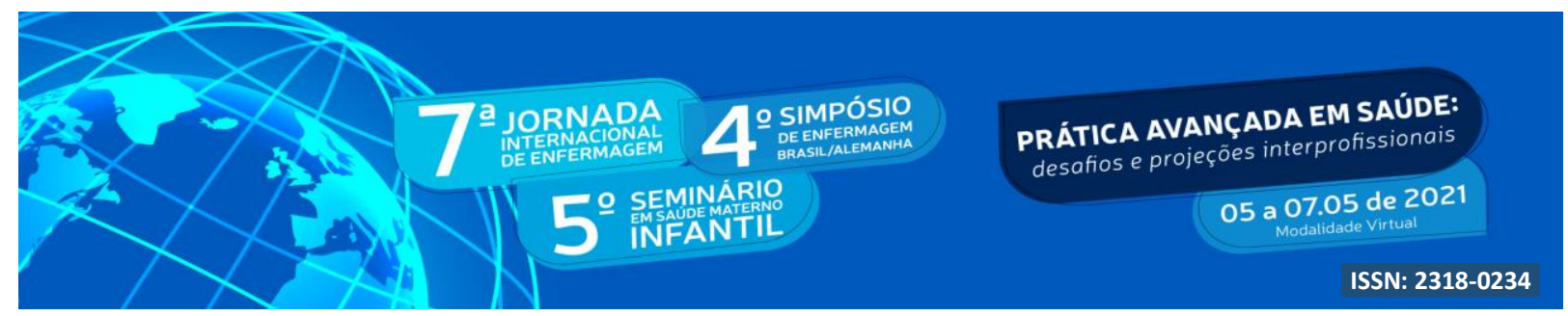

O primeiro encontro da disciplina ocorreu de forma presencial, anterioremente à pandemia, no mês de março de 2020, momento em que foi solicitado para os profissionais que descrevessem o seu conceito individual de saúde, o primeiro solicitado. Como parte da dinâmica proposta, os profissionais não poderiam utilizar algumas palavras no conceito, orientação que acompanhou a elaboração até a versão final, sendo estas: qualidade, integridade, qualidade de vida, subjetividade e humanização.

Na retomada da disciplina de forma remota (online), ao longo do período de agosto à outubro de 2020, os profissionais realizaram leituras individuais e discussões grupais, que embasaram a construção de um segundo conceito, utilizando os autores: Camargo (2015), Canguilhem (2012) e um autor de escolha do pós-graduando que neste estudo foi Forget e Lebel (2001). Posteriormente, após a leitura e socialização grupal de Marx (2017), Guattari (2012), CNDSS (2008) e de mais dois autores complementares escolhidos pela autora, que neste estudo são Leff (2012) e Scliar (2007), foi elaborado o terceiro conceito, aqui apresentado. A cada encontro, os profissionais realizavam uma síntese sobre a leitura indicada, que era encaminhada às docentes da disciplina.

\section{RESULTADOS E DISCUSSÃO}

A partir da metodologia proposta pela disciplina, foram construídos dois conceitos de saúde: o individual inicial e o individual ampliado e inédito. O conceito de saúde inicial foi elaborado de forma individual, sem leitura prévia e com limitação do uso de algumas palavras. Já o conceito de saúde ampliado e inédito, foi construído de forma individual, mas com a colaboração do grupo, uma vez que as leituras propostas pela disciplina e as interações grupais influenciaram na sua composição. A seguir apresentamos os dois conceitos:

Conceito de saúde inicial: Saúde é o relativo bem-estar pessoal, incluindo aspectos físicos, biológicos, emocionais e ambientais.

Conceito de saúde ampliado e inédito: Saúde é o relativo bem-estar pessoal, caracterizado pelo conforto emocional resultante das relações respeitosas, empáticas e de gratidão estabelecidas com o meio em que vivemos. Refere-se ainda, à capacidade de reação às alterações das normas estabelecidas, adaptando-se às novas normas biológicas, permitindo 


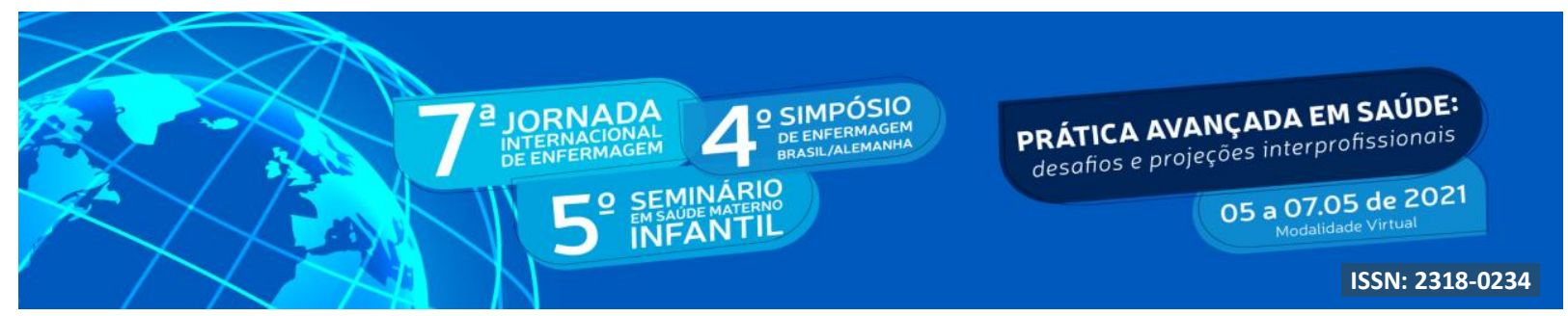

o equilíbrio entre a pessoa e o seu ambiente para que o progresso de ambos ocorra de forma sustentável. Saúde é a manutenção de relações saudáveis que suavizem as transformações da atuação humana na natureza, uma vez que a principal ação humana que interfere nas condições de saúde é o trabalho, é fundamental o equilíbrio para que essa relação seja sustentável e saudável, uma vez que ele impulsiona, regula e controla a natureza. A saúde é encontrada na singularidade, através da libertação dos rótulos produzidos em massa, que produzem indivíduos serializados e modelados, que replicam o conceito de bem-estar absoluto, engessado e inatingível. Para que a dimensão global de saúde seja alcançada, além das perspectivas singulares é necessário que os determinantes de saúde sejam direcionados às coletividades, às suas necessidades resultantes das interações com o meio, provendo a equidade no acesso e na utilização de serviços de saúde eficientes. Saúde é vida produtiva, é dignidade para as pessoas como cidadãos e seres humanos, é considerar as distintas adversidades que envolvem as condições humanas a partir de uma visão holística.

A partir das leituras e interações grupais, percebemos o quanto o conceito inicial de saúde é limitado e que vários prismas possibilitam a expansão do olhar sobre o mesmo. Entende-se que para além de expandir um conceito, podemos qualificar as práticas profissionais quando embasamos as ações e serviços de saúde na literatura científica e diversa existente, incluindo as singularidades e subjetividades dos indivíduos e coletividades.

A validação dos sentimentos, das subjetividades, das emoções e pequenas belezas do cotidiano são expressões da felicidade e para Camargo (2015) são possibilidades de promoção da saúde. Segundo o autor, saúde e felicidade estão intimamente ligados, entendendo a saúde não como o perfeito estado físico e sim como o estado de satisfação pessoal consigo e com o meio e que está inserido.

Essa visão singular de saúde, em partes, equivale ao conceito de Canguilhem (2012), que entende a saúde como um estado de adaptação. Ou seja, o organismo que é capaz de superar uma norma estabelecida e reestabelece o funcionamento a partir de uma nova norma é saudável. Assim, o autor também considera que a saúde é um estado individual, relativo, influenciado pela interação com o meio e não absoluto.

O equilíbrio entre o meio e as pessoas, a partir de interações sustentáveis é a ideia de saúde expressa pela abordagem ecossistêmica. Ela inclui os seres humanos como parte 


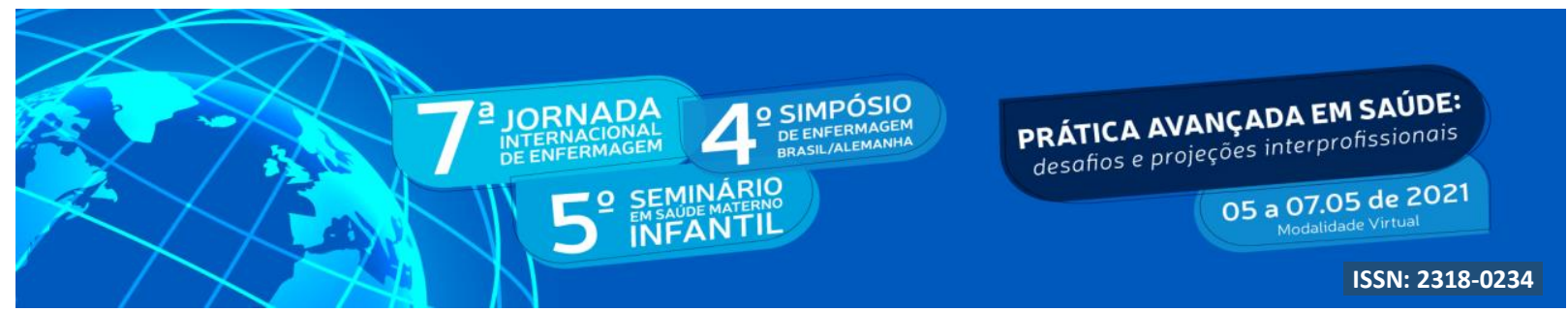

integrante dos ecossistemas, relacionando a saúde das pessoas aos demais organismos. Nessa perspectiva, a promoção de saúde é proposta através da gestão criteriosa do ecossistema, por meio da estabilização das atividades humanas no ambiente (FORGET, LEBEL, 2001).

Dentre as atividades humanas que influenciam diretamente na saúde das pessoas e o ambiente, o trabalho é a ação humana que impulsiona, regula e controla a natureza (MARX, 2017). E a qualidade de vida, condição básica para manutenção da saúde, está relacionada com a possibilidade de prover as suas necessidades através do trabalho, porém com o mundo capitalista e conectado em que vivemos, a busca pela inclusão nas modernidades capitalísticas pode gerar sofrimento, devido à busca incessante pela inserção nos moldes. Essa modernidade é composta por uma variedade de máquinas que produzem indivíduos modelados, registrados e produzidos em série e massa (GUATTARI, 2005).

A modernidade que produz indivíduos modelados, influencia a qualidade e quantidade de vida das pessoas, pois a busca incessante pelas necessidades vendidas pelas mídias desequilibra a sustentabilidade das relações. Sabendo que a saúde está intimamente ligada à qualidade de vida, é necessário considerar as necessidades e valores individuais, estabelecendo um processo de reapropriação das condições de vida como um valor fundamental (LEFF, 2012). A qualidade de vida, depende então do equilíbrio e da sustentabilidade, ela engloba a vida e o cotidiano das pessoas, em seus diversos determinantes.

Nesse contexto, os Determinantes Sociais em Saúde (DSS) são fundamentais para o direcionamento das práticas profissionais a partir da perspectiva ampliada de saúde. Os DSS possibilitam a promoção da saúde global pois conectam diferentes fatores que influenciam na saúde das pessoas, através da abordagem de questões sociais, laborais, econômicas e ambientais (CNDSS, 2008). A saúde apresenta uma representatividade diferente para cada indivíduo, dependendo da época, local, classe social, valores individuais, concepções científicas, religiosas, filosóficas e crenças. Dessa forma, entende-se a necessidade de conceituar a saúde a partir de uma visão holística, que contemple as distintas adversidades que envolvem a condição humana. (SCLIAR, 2007). 


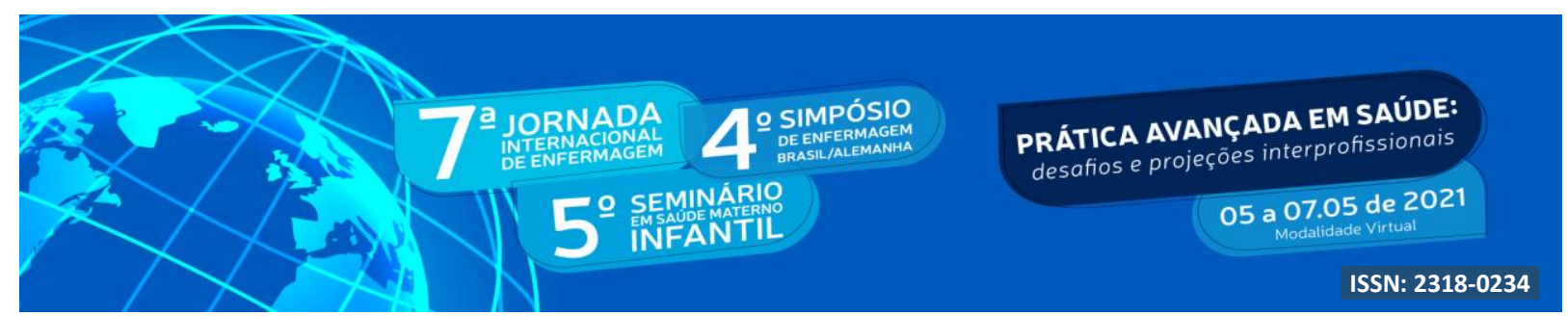

\section{CONCLUSÃO}

O processo de criação deste estudo, através da interação grupal de profissionais pósgraduandos de distintos núcleos profissionais, possibilitou a expansão do olhar sobre o conceito de saúde e a reflexão do quanto ainda estamos imersos nas ideias de saúde como estado de perfeição. As leituras permitiram o embasamento das discussões, bem como o conhecimento de novas possibilidades do entendimento de saúde.

Após a conclusão das atividades, entende-se que devido as enormes iniquidades sofridas pela população mundial na atualidade, o conceito de saúde como o estado de completo bem-estar é inatingível e impossível de ser mantido de forma integral. Portanto, os profissionais de saúde devem estimular um processo reflexivo das pessoas e comunidades, para que encontrem o seu estado de saúde, que possam viver com qualidade a partir da sua realidade, estabeleçam relações saudáveis entre si e o ambiente em que estão inseridos, que entendam que a saúde está ligada à felicidade e que ela está nas coisas simples e não nas ações vendidas elas mídias modernas.

Nesse contexto, o conceito ampliado de saúde pode contribuir para o entendimento de saúde como um aspecto global, influenciado pelas relações saudáveis e sustentáveis que considerem os fatores sociais, econômicos, laborais, emocionais, singulares. E pode contribuir ainda para o fomento de estratégias de promoção de saúde que não busquem a completude e sim a adaptação e a valorização das coisas simples que promovem a felicidade das pessoas e comunidades nas suas singularidades. Entende-se ainda que a reflexão sobre o conceito de saúde, objetivando a ampliação do olhar em relação aos seus determinantes pode contribuir para práticas profissionais de atenção integral à saúde das pessoas.

\section{REFERÊNCIAS}

1. BRASIL, Senado Federal. Constituição da República Federativa do Brasil. Brasília: Senado Federal, Centro Gráfico, 1988.Disponível em: http://www.planalto.gov.br/ccivil_03/Constituicao/CConstituiçao.htm. Acesso em: 18 de mar. de 2021. 


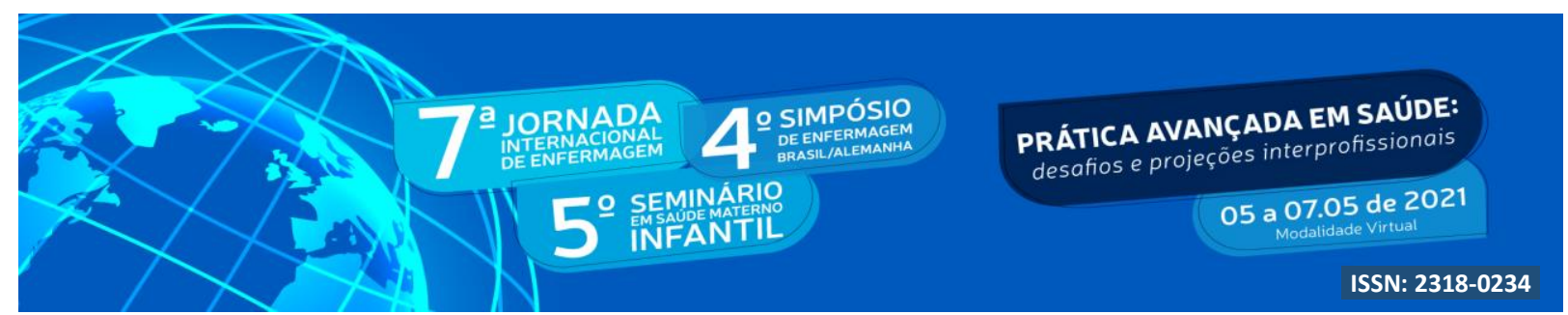

2. CAMARGO, J. J. Do que você precisa para ser feliz? $1^{\circ}$ ed. Porto Alegre, RS:L\&PM, 2015.

3. CAnguilhem, G. O normal e o patológico. $7^{\mathrm{a}}$ ed. Rio de Janeiro: ForenseUniversitária, 2012..

4. CNDSS-Comissão Nacional sobre Determinantes Sociais da Saúde. As causas sociais das iniqüidades em saúde no Brasil. Rio de Janeiro: Editora FIOCRUZ; 2008.

5. FORGET, G.; LEBEL, J. Ecosystem approach to human health: International journal of occupational and environmental health, supplement to v. 7, no. 2, Apr./June, 2001.

6. GUATTARI, F.; ROLNIK, S. Micropolítica - cartografias do desejo. $11^{\circ}$ edição. Petrópolis RJ: Editora Vozes; 2011.

7. LEFF, E. Saber ambiental: sustentabilidade, racionalidade, complexidade, poder. $9^{\mathrm{a}}$ ed. Petrópolis, RJ: Vozes, 2012.

8. MARX, K. O capital: crítica da economia política. v. 1. São Paulo: Nova Cultural, 1996.

9. WHO. World Health Organization. Constitution of World Health Organization. Geneva: WHO; 1948.

10. SCLIAR, M. História do conceito de saúde. Physis: Revista de saúde coletiva, v. 17, n. 1, p. 29-41, 2007. 